\title{
Primary Lens Luxation in Australian Tenterfield and Miniature Bull Terriers is Due to An Old ADAMTS17 Mutation and is an Additive Trait
}

\author{
Puya Gharahkhani ${ }^{1}$, Caroline O'Leary ${ }^{2, *}$, David Duffy $^{3}$, Michael Bernays ${ }^{4}$ and Myat Kyaw-Tanner ${ }^{1}$
}

\author{
${ }^{I}$ School of Veterinary Science, The University of Queensland, Gatton, QLD, 4343, Australia; ${ }^{2}$ Centre for Companion \\ Animal Health, The University of Queensland, Brisbane, QLD, 4072, Australia; ${ }^{3}$ Queensland Institute of Medical \\ Research, Brisbane, QLD, 4029, Australia; ${ }^{4}$ Animal Eye Services Logan Road, Underwood, QLD, 4119, Australia
}

\begin{abstract}
Primary Lens Luxation (PLL) is an inherited disease common in Terrier breeds. A truncating mutation in the ADAMTS17 orthologue on CFA03 is reported to cause PLL in 17 breeds, mostly Terriers. This study investigated the mode of inheritance and penetrance of PLL, age of the ADAMTS17 mutation, and other possible causes of PLL in Australian Tenterfield Terriers and Miniature Bull Terriers. The ADAMTS17 mutation and 30 nearby microsatellites on CFA03 were genotyped in 66 Australian Tenterfield Terriers, and 74 Miniature Bull Terriers. Lifetime risk of developing clinical PLL in homozygote and heterozygous animals, penetrance of PLL in general population, and recurrence risk of the disease for offspring and siblings of an index case was estimated. The effect of the ADAMTS17 mutation on the risk of PLL was dose and age dependent, and best fitted an additive model. Primary lens luxation was fully penetrant in homozygotes over 6 years and incompletely penetrant with a significant risk of PLL in heterozygote animals. Microsatellite haplotype testing estimated the mutation to be an old mutation, consistent with its appearance on several different background haplotypes and suggesting it entered the tested Tenterfield Terrier population at least three times, and the tested Miniature Bull Terrier population once. Finite polygenic model and survival analyses suggested that the ADAMTS17 mutation is the main cause of PLL in these breeds, and if the PLL phenotype is controlled by factors other than this mutation, such as a second locus or an environmental effect, the effect is much less than that of this disease associated SNP.
\end{abstract}

Keywords: ADAMTS17 mutation, Miniature Bull Terrier, Mode of inheritance, Penetrance, Primary Lens Luxation, Tenterfield Terrier.

\section{INTRODUCTION}

Primary Lens Luxation (PLL) is an inherited disease in which the lens comes free from its attachments and moves anteriorly or posteriorly in the eye. In Tibetan Terriers, Miniature Bull Terriers, and Shar Peis, lens luxation is due to an abnormal arrangement of the zonular fibres of the ciliary processes, which hold the lens in its normal position in the eye $[1,2]$. The disease is common in Terrier breeds such as the Tibetan Terrier, Miniature Bull Terrier and Jack Russell Terrier; but has also been reported in non-Terrier breeds such as the Chinese Shar Pei and Australian Cattle Dog. Primary Lens Luxation is usually first diagnosed in dogs when they are 2-6 years old, with the average age being 4.7-5.2 years $[3,4]$.

A truncating mutation in the canine ADAMTS17 orthologue [Entrez Gene ID: 488708] was recently shown by ourselves and others to cause PLL in Jack Russell Terriers, Miniature Bull Terriers and Lancashire Heelers [5]. This mutation has been also identified in 14 additional breeds,

*Address correspondence to this author at the Centre for Companion Animal Health, The University of Queensland, Brisbane, QLD, 4072, Australia; Tel: 61 (0)7 3365 2122; Fax: 61 (0)7 3346 9822;

E-mail: c.oleary@uq.edu.au mostly Terriers or breeds with Terrier co-ancestry, but also in some breeds with more diverse origins such as the Australian Cattle Dog. However, this mutation was not detected in PLL affected animals in some other breeds including the Shar Pei and Brittany Spaniel [6]. This GT to AT splice donor site mutation located at the 5' end of intron 10 , results in the skipping of exon 10 during transcription, a shift in the normal reading frame and a premature stop codon. Primary lens luxation is reported to have an autosomal recessive mode of inheritance in these breeds. However in Farias' 2010 study, 23 of 196 dogs with PLL were heterozygous for the ADAMTS17 mutation, and 12 were homozygous for the normal allele [5]. Gould et al., also suggested an increased but low risk of PLL in $A / G$ heterozygote animals [6].

The mode of inheritance and penetrance of the ADAMTS17 mutation, and whether other genetic or environmental factors contribute to the development of clinical PLL is not well known yet. The aim of this study was to further investigate the mode of inheritance and penetrance in PLL using ADAMTS17 mutation data, estimate the age of the mutation, and to investigate evidence for other genetic and environmental factors affecting the expression of the ADAMTS17 mutation in Australian populations of 
Tenterfield Terrier and Miniature Bull Terriers. Here we report that the ADAMTS17 mutation has a dose and age dependent penetrance, and an additive mode of inheritance in Australian Miniature Bull Terriers and Tenterfield Terriers. It is the main cause of PLL in those breeds, and is probably an old mutation.

\section{MATERIAL AND METHODS}

\section{Animal Ethics}

The University of Queensland Animal Ethics Committee approved this study SVS/089/907[nf], SVS/095/08, SVS/ 284/10/QCCC/ACAHF/KIBBLETRUST (NF), as did The University of Queensland Human Ethics Committee 2007000223.

\section{SELECTION OF DOGS AND gDNA EXTRACTION}

Sixty-six Tenterfield Terriers and 74 Miniature Bull Terriers from a population in which PLL was segregating were enrolled in the study. Veterinary ophthalmologists examined the dogs' eyes after pupillary dilation with $1 \%$ Topicamide (Alcon-Couvreur N.V., Puurs, Belgium) and using indirect ophthalmoscopy, applanation tonometry and slit lamp biomicroscopy. Diagnostic criteria included any evidence of PLL including the presence of an aphakic crescent, iridonesis, elevated intraocular pressure, anteriorly displaced vitreous or vitreous syneresis. Pedigrees were collected if available. Genomic DNA was extracted from EDTA blood using a salt extraction protocol [7], and QIAamp DNA Mini Kit (QIAGEN, Hilden, Germany) according to manufacturer instructions.

\section{GENOTYPING THE ADAMTS17 MUTATION} [5].

Genotypes for the ADAMTS17 SNP were performed as in

\section{GENOTYPING MICROSATELLITES ON CFA03}

Thirty microsatellites on CFA03 were amplified. Thirteen of these microsatellites were located in the region between $40 \mathrm{Mb}$ to $45 \mathrm{Mb}$, the region containing the canine ADAMTS17 mutation which is located at $43.52 \mathrm{Mb}$. Eight of 30 were from a previous study [8], and 22 were from the available databases [9-12] (Supplementary Material 1). Microsatellites were amplified using primers fluorescently labelled using a M13 tailing method [13]. All microsatellites were initially amplified in singleplex PCR and results compared with those obtained from multiplex PCR to ensure consistency. Singleplex PCRs contained 10-30 ng DNA, $1 \mathrm{x}$ PCR Buffer, $0.2 \mu \mathrm{M}$ of each dNTP, $0.05 \mu \mathrm{M}$ forward and $0.1 \mu \mathrm{M}$ reverse primers, $0.1 \mu \mathrm{M}$ labelled M13 primer, and 1 U HotstarTaq ${ }^{\circledR}$ DNA Polymerase (QIAGEN, Hilden, Germany) in a $10 \mu 1$ reaction volume. The M13 primer was labelled with 6FAM ${ }^{\mathrm{TM}}$, VIC®, NED ${ }^{\mathrm{TM}}$ and PET® dyes (DS$33 \mathrm{GeneScan}^{\mathrm{TM}}$ Installation Standard, Applied Biosysyems, Foster City, USA). Microsatellites were amplified at $95^{\circ} \mathrm{C}$ for $15 \mathrm{~min}$; followed by 35 cycles of $94^{\circ} \mathrm{C}$ for $1 \mathrm{~min}, 55^{\circ} \mathrm{C}$ for 1 $\mathrm{min}$, and $72^{\circ} \mathrm{C}$ for $1 \mathrm{~min}$; and one cycle of $72^{\circ} \mathrm{C}$ for $10 \mathrm{~min}$.

Seven multiplex PCRs were performed (Supplementary Material 1). Ten $\mu$ l PCRs contained 10-30 ng DNA, 1x Multiplex PCR Master Mix (QIAGEN, Hilden, Germany), variable concentrations of forward and reverse primers, and $0.2-0.3 \mu \mathrm{M}$ of each labelled M13 primer. The M13 primer was labelled with $6 \mathrm{FAM}^{\mathrm{TM}}$, VIC $®$, NED ${ }^{\mathrm{TM}}$ and PET $\AA$ dyes. Microsatellites were amplified at $95^{\circ} \mathrm{C}$ for $15 \mathrm{~min}$, followed by 35 cycles of $94^{\circ} \mathrm{C}$ for $30 \mathrm{~s}, 55^{\circ} \mathrm{C}$ for $90 \mathrm{~s}$, and $72^{\circ} \mathrm{C}$ for 1 min; and one cycle of $72^{\circ} \mathrm{C}$ for $10 \mathrm{~min}$. Labelled PCR products were genotyped using 3130XL Genetic Analyzer (Applied Biosystems, Foster City, USA), and analysed with software Genemapper version 3.7 (Applied Biosystems, Foster City, USA).

\section{LINKAGE AND ASSOCIATION ANALYSES}

Sib-pair64 software [14] was used for non-parametric linkage analysis and also association analysis between the microsatellites and the PLL phenotype, and to determine segregation of PLL linked markers with the ADAMTS17 mutation. Superlink1_7.linux32 [15] was also used for parametric linkage analysis using microsatellites and disease phenotype data.

\section{INVESTIGATION OF MODE OF INHERITANCE AND DISEASE PENETRANCE}

Kruskal Wallis testing for mean age at diagnosis was performed using Sib-pair64 software to investigate the effect of different genotypes of the PLL causative ADAMTS17 biallelic SNP on the age of onset of disease. Survival analysis was performed using the Kaplan Meier method, Weibull model, and gamma distribution parametric model in R-2.10.0 software [16]. Single major locus recurrent risk was calculated using Sib-pair64 software, both to further investigate the mode of inheritance and penetrance of PLL, as well as to evaluate the risk of recurrence and risk to offspring from varying types of matings between affected and unaffected animals.

\section{ESTIMATION OF THE AGE OF THE ADAMTS17 MUTATION}

Superlink1_7.linux32 was used to analyse microsatellite haplotypes to allow estimation of the age of the ADAMTS17 mutation. This calculation was based on the number of recombination events in the haplotypes. The DMLE programme (Version 2.3) [17] was also used to estimate the age of the ADAMTS17 mutation, based on the recombination pattern observed in the flanking microsatellite markers.

\section{INVESTIGATION OF CAUSES OTHER THAN THE ADAMTS17 MUTATION IN PLL}

Finite polygenic model analysis using Sib-pair64 and survival analysis using R-2.10.0 were performed to investigate the effect of factors of than the ADAMTS17 mutation in PLL.

\section{RESULTS}

\section{PLL Status}

Sixty-five Tenterfield Terriers and 65 Miniature Bull Terriers had clinical PLL phenotype recorded as diagnosed by a veterinary ophthalmologist, with 10 additional animals (1 Tenterfield and 9 Miniature Bull Terriers) having no phenotype recorded. 


\section{PLL PHENOTYPE WAS LINKED TO MICRO- SATELLITES ON CFA03}

Parametric linkage analysis supported linkage between 39.05 - 48.6 Mb on CFA03 and the PLL phenotype in both breeds. Maximum LOD scores obtained using dogs of both breeds in the analysis were 1.54 for CamC0.045 (43.5 Mb on CFA03), and 1.40 for ZUBECA4 (42.8 Mb on CFA03). Similarly, non-parametric linkage analysis also supported linkage between 41.5 - 45.3 Mb on CFA03 and PLL in both breeds, with a maximum LOD score of 1.46 for CamC0.045 and ZUBECA4. Association analysis also showed that allele 422 of CamC0.045 was associated with PLL (empirical $\left.P=2 \times 10^{-6}\right)$.

\section{SEGREGATION OF LINKED MICROSATELLITES AND THE $A D A M T S 17$ MUTATION}

Association analysis showed the ADAMTS17 SNP allele $A$, consistently segregated with CamC0.045 allele 422 (LD $\left.r^{2}=0.96\right)$. The frequency of the $A-422$ haplotype in the study population was 0.41 (0.38 in the Tenterfield Terrier samples, 0.51 in the Mini Bull Terrier samples).

\section{MODE OF INHERITANCE AND PENETRANCE OF PLL}

The prevalence of clinical PLL in this population of Tenterfield Terriers was $12 \%(8 / 65)$, and $23 \%(15 / 65)$ in Miniature Bull Terriers. Altogether, 23 dogs had clinical PLL with 15 being $A / A$ (65\%) for the ADAMTS17 SNP and 8 being $A / G(35 \%)$. While $7.5 \%$ of clinically unaffected animals were $A / A$, all $A / A$ dogs over 6 years old had clinical PLL (Table 1).
Kruskal Wallis testing showed significant differences between groups of animals with different PLL status according to their ADAMTS17 SNP genotype $(P=0.0016)$ (Table 2). Twenty-three dogs in this study were $A / A$ homozygotes, with $8 \mathrm{dogs}$ clinically unaffected by PLL. However, all of these $A / A$ unaffected dogs were young. The mean age of $A / A$ unaffected animals was 21 months, whereas the mean age of $A / A$ affected animals was 54 months.

The mean age of diagnosis with PLL was 54 months in $A / A$ homozygote animals and 72 months in $A / G$ heterozygote animals. The mean age of $A / G$ heterozygotes with no clinical signs of PLL was 49 months, compared with 72 months in heterozygous animals with clinical PLL.

Survival analysis using non-parametric Kaplan-Meier estimate analysis showed that the estimated lifetime risk of clinical PLL for $A / A$ homozygotes was higher than in $A / G$ heterozygote dogs (Fig. 1). No dogs were diagnosed with PLL at 2 years of age, but by 3 years of age PLL had been diagnosed in $10 \%$ of $A / A$ homozygotes. Homozygous $A / A$ dogs over 6 years of age showed $100 \%$ disease penetrance. In heterozygote animals, penetrance of the disease phenotype was less than 5\% in animals aged 3 years, and $60 \%$ in animals aged 10 years (Fig. 1). There was zero penetrance in $G / G$ homozygote dogs in the study population. Survival analysis using the parametric Weibull model produced similar results to those obtained from non-parametric KaplanMeier estimate analysis (data not shown). Ascertainment corrected survival analysis under a Gamma distribution parametric model also gave similar results to those from the nonascertainment corrected Kaplan-Meier and Weibull model analysis (data not shown), so we believe our penetrance estimates to be robust to model misspecification.

Table 1. Proportions of ADAMTS17 SNP Genotypes in PLL Clinically Affected and Clinically Unaffected Dogs (Miniature Bull Terriers and Tenterfield Terriers Combined)

\begin{tabular}{|l|l|l|l|}
\hline ADAMTS17 SNP Genotype/PLL Status & $\boldsymbol{A} / \boldsymbol{A}$ & $\boldsymbol{A} / \boldsymbol{G}$ & $\boldsymbol{G} / \boldsymbol{G}$ \\
\hline \hline PLL affected & $65.2 \%(15 / 23)$ & $34.8 \%(8 / 23)$ & $0 \%(0 / 23)$ \\
\hline PLL unaffected (all ages) & $7.5 \%(8 / 107)$ & $47.7 \%(51 / 107)$ & $44.8 \%(48 / 107)$ \\
\hline PLL unaffected > 6 years & $0 \%(0 / 21)$ & $61.9 \%(13 / 21)$ & $38.1 \%(8 / 21)$ \\
\hline
\end{tabular}

Table 2. Kruskal Wallis Testing for Mean Age at First Diagnosis of Clinical PLL

\begin{tabular}{|l|l|l|l|}
\hline $\begin{array}{l}\text { PLL Status/ ADAMTS17 } \\
\text { SNP Genotypes * }\end{array}$ & $\begin{array}{l}\text { Number of } \\
\text { Dogs }\end{array}$ & $\begin{array}{l}\text { Mean age of Diagnosis in PLL Affected and } \\
\text { Unaffected Dogs }\end{array}$ & $\begin{array}{l}\text { Standard Deviation of Mean Age of Diagnosis in } \\
\text { PLL Affected and Unaffected Dogs }\end{array}$ \\
\hline \hline Unaffected-A/A & 8 & 21 & 16 \\
\hline Unaffected-A/G & 51 & 49 & 33 \\
\hline Unaffected- $G / G$ & 48 & 47 & 33 \\
\hline Affected-A/A & 15 & 54 & 11 \\
\hline Affected- $A / G$ & 8 & 72 & 28 \\
\hline
\end{tabular}

This test compared the mean age of diagnosis of clinically affected and unaffected animals according to their genotypes for the ADAMTS17 SNP $(P=0.0016)(4$ degrees of freedom and $\mathrm{H}=17.45$ ). The $P$ value indicates that the differences between mean ages are significant between different groups of animals according to their PLL status and ADAMTS17 SNP genotypes.

$* A=$ mutant allele causing PLL in Miniature Bull Terriers and Tenterfield Terriers, $G=$ wild type allele 


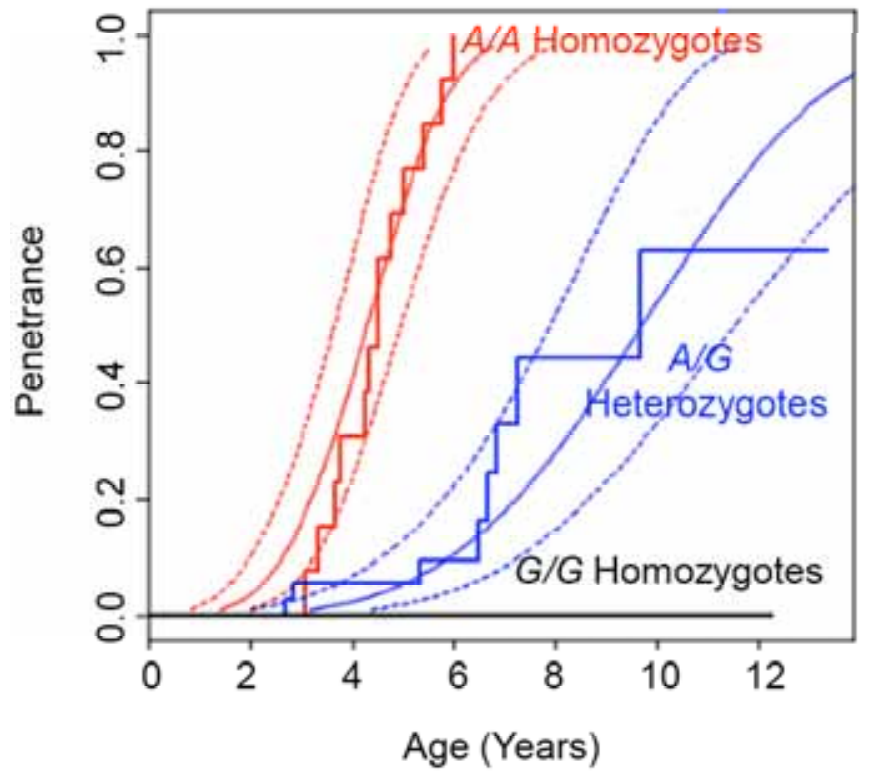

Fig. (1). Penetrance of the ADAMTS17 SNP in the clinical phenotype calculated using age at first diagnosis. This plot shows survival analysis curves created by R-2.10.0 software, reflecting the lifetime risk of developing PLL due to the ADAMTS17 mutation in years of age at the time of first diagnosis. This plot was produced using the non-parametric Kaplan-Meier estimate and parametric Weibull model. Step curves are the empirical age at onset curves, created using non-parametric Kaplan-Meier estimate analysis. The continuous lines show the lifetime risk estimates obtained using Weibull model survival analysis in $A / A$ homozygote animals (red line), $A / G$ heterozygote animals (blue line) and $G / G$ homozygote animals (black horizontal line). Maximum and minimum $95 \%$ confidence intervals for age-at-first diagnosis created using Weibull model analysis are shown with non-continuous curves.

Only $10 \mathrm{~A} / \mathrm{G}$ heterozygote animals were over 7 years old in the study population, resulting in a wide estimated confidence interval for these animals (Fig. 1). Therefore, for subsequent risk calculations, a penetrance value of $45 \%$ was used, which was the value calculated from dogs up to seven years of age. Under this model (mutation frequency 0.41 , lifetime penetrance $100 \%, 45 \%, 0 \%$ ), the recurrence risk to offspring and siblings of an index case was predicted to be 0.53 (see Table 3).

Table 3. Estimated Risk to Offspring from Various Mating Types, for the Development of Clinical PLL

\begin{tabular}{|l|l|l|}
\hline Mating & $\begin{array}{l}\text { Proportion of Offspring } \\
\text { from Matings }\end{array}$ & $\begin{array}{l}\text { Risk to Offspring of } \\
\text { Clinical PLL }\end{array}$ \\
\hline \hline UnA $^{1} x$ UnA & 0.67 & 0.12 \\
\hline Aff $^{2}$ x UnA & 0.29 & 0.26 \\
\hline Aff $x$ Aff & 0.04 & 0.57 \\
\hline
\end{tabular}

This table shows the estimated proportion of offspring with clinical PLL from various types of matings, obtained by single major locus recurrent risk calculation using Sibpair64 .

${ }^{1}$ UnA; clinically unaffected by PLL

${ }^{2}$ Aff; clinically affected by PLL

\section{ESTIMATION OF THE AGE OF THE ADAMTS17 SNP}

Eight of 15 Miniature Bull Terriers with clinical PLL were homozygous and seven were heterozygous for the disease associated SNP allele, and nine of these animals shared a common 9.6 Mb haplotype, with a core $600 \mathrm{~Kb}$ haplotype consisting of the ADAMTS17 SNP and two of the closest flanking microsatellite markers shared by all except one animal (where there was an apparent mutational event). The common extended haplotype was also seen in some affected Tenterfield Terriers, so is likely to represent the ancestral background haplotype. The other risk haplotypes were consistent with a pattern of six ancestral recombination events in this 9.6 Mb interval. Using the DMLE+ program to analyse these data, we estimated the age of the mutation at 26 (21-28) generations old.

Similar haplotype analysis in Tenterfield Terriers in the same linked region showed that 7 of 8 animals with clinical PLL were homozygous for allele 422 at the nearest marker CAMC03.045. However, unlike the Miniature Bull Terriers, haplotype homozygosity was not present in the entire region. Eighteen PLL associated haplotypes were identified in this region, all containing allele 422 . Three of these haplotypes were major haplotypes, with the other 15 variants likely to have occurred with recombination events within these main haplotypes. Despite this, the estimated age of the mutation was not greatly different, at 27 (23-29 generations).

Given that there are multiple obligate recombination events in the $600 \mathrm{~Kb}$ interval between the two nearest flanking markers to the ADAMTS17 mutation, this precludes the mutation being young. This is also implied by the same mutation being present in multiple dog breeds [5]. The estimated mutation age is quite sensitive to assumptions about how rapidly the breeds have expanded in size: 76 generations (95\% CI, 53-118), assuming the breeds expanded at $10 \%$ per generation, and 23 generations (95\%CI, 25-73) assuming $25 \%$ expansion per generation. Based on breed records, the latter expansion rate seems more plausible [18]. Assuming the mutation is relatively neutral with respect to reproductive fitness, and has not been actively selected for as a correlated trait by breeders, we can also estimate mutation age based on the mutation frequency. We used effective population sizes of 50 and 100 animals, based on those estimated for Terrier breeds in Australia [19]. Under the same expansion estimates, we obtain estimates of 10 generations (25\% expansion) and 24 generations (10\% expansion). Overall, these analyses would suggest that this mutation entered the Australian population base early in the twentieth century.

\section{INVOLVEMENT OF OTHER GENETIC OR NONGENETIC FACTORS IN PLL}

Finite polygenic model analysis when combining data from both breeds indicated that additive quantitative trait locus variance and additive polygenic variance decreased after including the ADAMTS17 SNP in the analysis (Table 4). This indicated that the ADAMTS17 mutation accounted for much of the PLL phenotype, decreasing the likelihood of involvement of a second locus in PLL.

Goodness of fit measures for mixed effects survival analysis models that excluded or included the ADAMTS17 
Table 4. The PLL Phenotype is Largely Due to the ADAMTS17 SNP as Determined Using the Finite Polygenic Model Analysis

\begin{tabular}{|l|l|l|}
\hline Variance/Likelihood & Without inclusion of ADAMTS17 SNP & With inclusion of ADAMTS17 SNP \\
\hline \hline Additive QTL ${ }^{1}$ variance & $18.328804(51.6 \%)$ & $0.146914(3.5 \%)$ \\
\hline Additive polygenic variance & $0.792643(9.8 \%)$ & $0.456353(11.7 \%)$ \\
\hline Environmental variance & $0.047852(38.6 \%)$ & $0.046973(84.7 \%)$ \\
\hline Modal model log likelihood & -504.372 & -391.767 \\
\hline Mean model log likelihood & -483.808 & -398.259 \\
\hline C.V. ${ }^{2}$ Log likelihood & $4.63 \%$ & $2.35 \%$ \\
\hline
\end{tabular}

This table shows the variance of the quantitative trait PLL in the combined Miniature Bull Terrier and Tenterfield Terrier populations with and without the effect of the ADAMTS17 SNP. Additive quantitative trait locus variance and additive polygenic variance decreased significantly after including the ADAMTS17 SNP, indicating that ADAMTS17 SNP is the main cause of PLL.

${ }^{1}$ Quantitative trait locus

${ }^{2}$ Coefficient of variation

SNP as a fixed effect, were not significantly different $(P=$ 1.0 for null, 0.878605 for integrated and 1.000 for penalised analysis), indicating that the ADAMTS17 mutation could account for most (or all) of the familial aggregation of the PLL phenotype.

\section{DISCUSSION}

Primary Lens Luxation is due to a mutation in the gene ADAMTS17 on CFA03 in 17 canine breeds, mostly Terriers and breeds with Terrier co-ancestry, but also in some breeds with more diverse origins [5, 6]. Despite this, the actual mode of inheritance of PLL remains unclear. The current study findings suggest PLL shows dose and age dependant effects, consistent with an additive allelic model. Thus on average, animals homozygous for the mutation are expected to show the disease earlier in life (mean age of 4-5 years) compared to heterozygous animals (mean age 6 years or more). Survival analysis showed that penetrance of the PLL mutation increased with age, with full penetrance in $A / A$ animals over 6 years, and incomplete but up to $60 \%$ penetrance in $A / G$ animals by 10 years of age. Survival analysis with and without ascertainment correction produced the same results, suggesting the penetrance value calculated for the PLL mutation is unlikely to be biased due to animal selection in this study.

In calculating the risk of disease in the general population, we used the penetrance estimate of $45 \%$ in heterozygous animals up to age 7 years. This was because there were only $10 \mathrm{~A} / \mathrm{G}$ heterozygote animals over the age of 7 years in our study population, resulting in a wide $95 \%$ confidence interval for $A / G$ heterozygote animals over this age. Due to the lack of published data on the actual prevalence of PLL in Miniature Bull Terriers or Tenterfield Terriers, the estimated risks for the disease in the general population are projected values.

In our recent study in Jack Russell Terriers, Miniature Bull Terriers and Lancashire Heelers, 9.4\% (23/196) of PLL clinically affected $\operatorname{dogs}$ were $A / G$ heterozygotes [5]. Thus in PLL in these breeds, the estimated penetrance for heterozygote animals is lower than expected in a purely additive codominant disease. However, most of the animals in this study were under 7 years, and so may develop disease with age. Gould et al., also suggested an increased but low risk of PLL in heterozygote animals [6]. However, in the current study, a penetrance of as high as $60 \%$ was calculated in heterozygous animals up to 10 years of age, and a penetrance of $100 \%$ in homozygous animals over the age of 6 years. This suggests that the mode of inheritance may best fit an additive model. Such modes of inheritance have been reported in inherited diseases such as Parkinsonism in humans [20].

Gene additive effects, gene interactions, and interactions between genes and the environment can also affect the mode of inheritance in genetic diseases. Thus, an alternate hypothesis to additive inheritance could be that a second or modifying locus is associated with PLL, or environmental factors could interact with genetic factors to alter penetrance and the clinical picture of disease.

One of the important results of this study was the relatively high risk of heterozygote animals developing clinical PLL, a disease which is commonly painful. Reducing the prevalence of clinical disease in recessive diseases may involve breeding carrier or affected dogs to unaffected homozygous dogs to produce unaffected homozygote and/or carrier pups. However, when carrier animals have a relatively high risk of developing a potentially painful disease this strategy may be problematic. Further, the frequency of the PLL disease allele in some populations may also be high, making removal of all PLL affected and carrier animals impractical in these breeds due to the resultant loss of genetic variability in the breed. Hence, control of PLL may include maintenance of as many breeding lines as possible, by breeding as many unaffected homozygous animals as possible to related breeds with a low PLL mutation prevalence, and screening the progeny for inherited diseases. This strategy allows breeding away from PLL whilst minimising the risk of unveiling other genetic problems.

In this study, calculations estimating the lifetime risk of developing clinical PLL in homozygous and heterozygous animals, the estimated penetrance of PLL in general population, and the recurrence risk for offspring and siblings 
of an index case were performed. These data provide clinicians with indications of the risk of, and likely clinical onset of, clinical PLL in both homozygote and heterozygote Miniature Bull Terriers and Tenterfield Terriers in Australia. It also identifies problems in breeding with animals carrying this mutation and proposes methods to address this issue.

In the Miniature Bull Terriers one main disease related haplotype was present in dogs in this study, supporting the age of the mutation in this population being approximately 100 years. Miniature Bull Terriers are believed to have their origins in the Old English White Terrier, the Dalmatian and the Bulldog, and were first documented as a breed in the late nineteenth century. As the Bulldog and Dalmatian have not been reported to suffer from PLL, the extinct Old English White Terrier could thus be the originating breed for the mutation in the Miniature Bull Terrier population in this study [21-23].

In the Tenterfield Terrier sample, we inferred that the mutation was introduced by multiple founders, each time with the disease associated mutation being on a different background haplotype. Maximum likelihood estimation of founder haplotypes is difficult because of the persistent linkage disequilibrium seen in our pedigree founders, so our conclusions should be a little tentative. In two older haplotypes the disease associated region had been reduced to a very small region around allele 422 , consistent with more recombination events occurring in many generations over a longer time frame. These haplotypes may have their origins in the founders of the Tenterfield Terrier breed in Australia, although the breed has only been registered, and so had a closed breeding pool, for approximately two decades [21]. The third haplotype in the Tenterfield Terrier population was the commonest haplotype in the affected Miniature Bull Terriers.

This study found that the ADAMTS17 mutation was the main cause of PLL in the tested populations. However, difficulties in determining the mode of inheritance for PLL suggest a possible role for other genetic or nongenetic factors in the disease phenotype. Such factors may include other ADAMTS17 alleles, a second mutation with a lesser effect on the disease phenotype or environmental effects. In support of this, clinical PLL has been reported in $G / G$ homozygote dogs in several breeds including Miniature Bull Terriers, Lancashire Heelers, Jack Russell Terriers, Tenterfield Terriers, Rat Terriers, Jagdterriers, and Wire-haired Fox Terriers, suggesting genetic heterogeneity is possible in these breeds [5, 6]. Further, Gould et al., reported that in 16 other breeds with PLL, the ADAMTS17 causative mutation was not present, suggesting a genetically distinct form of the disease is possible in those breeds [6].

As the previous study reporting the PLL associated mutation in the canine ADAMTS17 gene did not sequence the introns and regulatory sequences [5], mutations in introns or regulatory sequences may not have been detected. Such sequence variants, as well as synonymous and non synonymous coding sequence changes, may affect the disease phenotype by altering RNA splicing or gene expression [24-26]. Thus, a hypomorphic allele of the ADAMTS17 SNP could be associated with a milder phenotype in compound heterozygotes, as evidenced by later disease onset and decreased penetrance. Hence, sequencing of ADAMTS17 introns, and 3' and 5' untranslated regions in dogs with varying clinical PLL status and PLL associated ADAMTS17 mutation genotypes may further our understanding of disease pathogenesis. Determination of ADAMTS17 expression levels using RT-PCR and protein quantitation on ocular tissues from animals with PLL could also provide evidence of involvement of various alleles of the ADAMTS17 SNP in PLL development.

Candidates for a modifying locus in PLL include an ADAMTSL gene, as encoded proteins from these genes have been suggested to interact with the ADAMTS proteins and have a regulatory effect on their activities [27]. Future GWAS using SNPs located throughout the whole canine genome may help to investigate the involvement of secondary or modifying loci in the development of PLL. However, an effect from environmental factors could also change the penetrance of the disease in $A / G$ heterozygote animals. Finally, it also may be speculated that a second mutation in the ADAMTS17 gene which is segregating with the PLL associated allele in heterozygote animals, could result in reduction of expression of the PLL associated allele, causing a milder phenotype in affected heterozygotes.

Mutations in ADAMTS17 in humans are associated with Weill-Marchesani syndrome (WNS) like syndrome, which is characterised by lens dislocation and short stature [28]. Interestingly, one of the reported mutations in humans with WMS-like syndrome is a homozygous $G>A$ splice-site donor mutation in intron 12 of the gene which produces a truncated transcript, similar to the mutation in the canine orthologue which causes PLL. In contrast, mutations in the coding regions of the ADAMTS17 gene were excluded from association with primary lens instability in the cat, and the FBN1 gene has been suggested as a potential candidate gene in this species [29], suggesting that genetic causes of inherited lens dislocation may be different in dogs and cats.

\section{CONCLUSIONS}

Inheritance of PLL in Australian Miniature Bull Terriers and Tenterfield Terriers is likely to most closely follow an additive model, with heterozygotes at significant risk for developing clinical PLL. The ADAMTS17 PLL associated SNP is likely to be an old mutation in the Terrier breeds, and has been introduced several times into the Australian Tenterfield Terrier population. If the PLL phenotype is controlled by factors other than the ADAMTS17 SNP, such as a second locus or an environmental effect, the effect is much less than that of this disease associated SNP. These findings support continued monitoring of carriers of the PLL mutation for evidence of lens luxation, as well as the mutation being an old one present in many dog breeds.

\section{CONFLICT OF INTEREST}

Declared none.

\section{ACKNOWLEDGEMENTS}

The authors thank Professor Gary Johnson for performing the ADAMTS17 mutation testing, Dr Cathryn Mellersh, and veterinary ophthalmologists Dr Edith 
Hampson, Dr Anna Deykin, Dr Mark Billson, Dr Anita Dutton, Dr Andrew Turner, Dr Bruce Robertson, Dr Rick Read, Dr Cameron Whittaker, and Dr Ziggy Chester. The authors would like to thank Dogs Queensland (previously The Queensland Canine Control Council) and the Dolby Bequest for funding.

\section{SUPPORTIVE/SUPPLEMENTARY MATERIAL}

Supporting information is available on the publishers Web site along with the published article.

\section{REFERENCES}

[1] Curtis R. Aetiopathological aspects of inherited lens dislocation in the Tibetan Terrier. J Comp Pathol 1983; 93(1): 151-63.

[2] Morris RA, Dubielzig RR. Light-microscopy evaluation of zonular fiber morphology in dogs with glaucoma: secondary to lens displacement. Vet Ophthalmol 2005; 8(2): 81-4.

[3] Ketteritzsch K, Hamann H, Brahm R, et al. Genetic analysis of presumed inherited eye diseases in Tibetan Terriers. Vet J 2004; 168(2): 151-9.

[4] Curtis R, Barnett KC, Lewis SJ. Clinical and pathological observations concerning the etiology of primary lens luxation in the dog. Vet Rec 1983; 112(11): 238-46.

[5] Farias FHG, Johnson GS, Taylor JF, et al. An ADAMTS17 splice donor site mutation in dogs with primary lens luxation. Invest Ophthalmol Vis Sci 2010; 51(9): 4716-21.

[6] Gould D, Pettitt L, McLaughlin B, et al. ADAMTS17 mutation associated with primary lens luxation is widespread among breeds. Vet Ophthalmol 2011; 14(4): 1-7.

[7] Miller SA, Dykes DD, Polesky HF. A simple salting out procedure for extracting DNA from human nucleated cells. Nucleic Acids Res 1988; 16(3): 1215.

[8] Sargan DR, Withers D, Pettitt L, et al. Mapping the mutation causing lens luxation in several terrier breeds. J Hered 2007; 98(5): 534-8.

[9] UniSTS. National Cener for Biotechnology Information. [cited $25^{\text {th }}$ Oct 2011]. Available from: http://www.ncbi.nlm.nih.gov/sites/ entrez?db=unists

[10] NHGRI Dog Genome Project. National Human Genome Research Institute. [cited $25^{\text {th }}$ Oct 2011]. Available from: http://research. nhgri.nih.gov/dog_genome/

[11] USCS genome bioinformatics: Dog (Canis lupus familiaris) Genome Browser Gateway. Genome Bioinformatics Group of UC Santa Cruz. [cited $25^{\text {th }}$ Oct 2011]. Available from: http://genome. ucsc.edu/cgi-bin/hgGateway.

[12] Ensembl: Dog. [cited 25 $5^{\text {th }}$ Oct 2011]. Available from: http:// ensembl.genomics.org.cn:8059/Canis_familiaris/Info/Index
[13] Oetting WS, Lee HK, Flanders DJ, et al. Linkage analysis with multiplexed short tandem repeat polymorphisms using infrared fluorescence and M13 tailed primers. Genomics 1995; 30(3): 450-8.

[14] Duffy's QIMR (Homepage on the internet). Duffy DL. Queensland Institute of Medical Research. [cited 25 $5^{\text {th }}$ Oct 2011]; Available from: http://genepi.qimr.edu.au/staff/davidD/\#sib-pair

[15] SUPERLINK version 1.7. Haifa: University of Technion. [updated $11^{\text {th }}$ Nov 2008; cited 25 ${ }^{\text {th }}$ Oct 2011]. Available from: http://cblfog.cs.technion.ac.il/superlink/

[16] The R Project for Statistical Computing. Institute for Statistics and Mathematics, WU Wien. [cited $25^{\text {th }}$ Oct 2011]. Available from: http://www.r-project.org/

[17] Reeve JP, Rannala B. DMLE+: Bayesian linkage disequilibrium gene mapping. Bioinformatics 2002; 18(6): 894-5.

[18] Slatkin M, Rannala B. Estimating the age of alleles by use of intraallelic variability. Am J Hum Genet 1997; 60(2): 447-58.

[19] Shariflou MR, James JW, Nicholas FW, Wade CM. A genealogical survey of Australian registered dog breeds. Vet J 2011; 189(2): 203-10.

[20] Klein C, Lohmann HK, Rogaeva E, Schlossmacher MG, Lang AE. Deciphering the role of heterozygous mutations in genes associated with parkinsonism. Lancet Neurol 2007; 6(7): 652-62.

[21] Australian National Kennel Council. Australian National Kennel Council Ltd. [cited $5^{\text {th }}$ Sep 2011]. Available from: http://www. ankc.org.au/

[22] American Kennel Club. American Kennel Club®. [cited $5^{\text {th }}$ Aug 2011]. Available from: http://www.akc.org/

[23] Breeds of Dogs, a reference to the world of dog's information [cited $5^{\text {th }}$ Aug 2011]. Available from: http://www.thebreedsofdogs. com/

[24] Chao HK, Hsiao KJ, Su TS. A silent mutation induces exon skipping in the phenylalanine hydroxylase gene in phenylketonuria. Hum Genet 2001; 108(1): 14-9.

[25] Montera M, Piaggio F, Marchese C, et al. A silent mutation in exon 14 of the APC gene is associated with exon skipping in a FAP family. J Med Genet 2001; 38(12): 863-7.

[26] Kimchi SC, Oh JM, Kim IW, et al. A "silent" polymorphism in the MDR1 gene changes substrate specificity. Science 2007; 315(5811): 525-8.

[27] Hirohata S, Wang LW, Miyagi M, et al. Punctin, a novel ADAMTS-like molecule, ADAMTSL-1, in extracellular matrix. J Biol Chem 2002; 277(14): 12182-9.

[28] Morales J, Al-Sharif L, Khalil DS, et al. Homozygous mutations in ADAMTS10 and ADAMTS17 cause lenticular myopia, ectopia lentis, glaucoma, spherophakia, and short stature. Am J Hum Genet 2009; 85(5): 558-68.

[29] Payen G, Hanninen RL, Mazzucchelli S, et al. Primary lens instability in ten related cats: clinical and genetic considerations. J Small Anim Pract 2011; 52(8): 402-10. 